\title{
Moraitis, Anastasia; Mavruk, Gülsah; Schäfer, Andrea \& Schmidt, Eva (Hg.) (2018): Sprachförderung durch kulturelles und ästhetisches Lernen. Sprachbildende Konzepte für die Lehrausbildung. Münster/New York: Waxmann
}

\author{
Jeannette Böttcher
}

Wie hängt Lehre mit Theater zusammen? Welche neuen Wege gibt es, um (Sprachen-)Lehren und Lernen zu fördern? Wie kann ich mein eigenes Lehren verbessern? Und wie kann ich meinen Schülern und Schülerinnen, aber auch meinen Lehramtsstudierenden bessere Lernmöglichkeiten bieten?

Diese Fragen sollten für jeden von Interesse sein, der lehrt und lernt, der sich immer wieder mit den komplexen Beziehungen zwischen (Fremd)Sprachenlehren und -lernen, zwischen kultureller Bildung und Integration, konfrontiert sieht.

Der Sammelband Sprachförderung durch kulturelles und ästhetisches Lernen - Sprachbildende Konzepte für die Lehrerausbildung der Herausgeberinnen Anastasia Moraitis, Gülsah Mavruk, Andrea Schäfer und Eva Schmidt präsentiert in elf Beiträgen unterschiedliche Kooperationsprojekte zum Thema kulturelle und ästhetische Bildung in vielschichtigen Lehr- und Lernszenarien. In Hinblick auf ästhetisches Lehren und Lernen bietet dieses Buch sowohl theoretische Grundlagen als auch konkrete Hinweise zur praktischen Umsetzung in alltäglichen Lehr- und Lernkontexten.

Die einzelnen Beiträge stellen nicht nur verschiedene Konzepte und Methoden von ästhetischem Lernen und (Fremd-)Sprachenerwerb bzw. Erziehung und Bildung vor, sondern differenzieren sie in ihren spezifischen Zielsetzungen und Potenzialen. Darüber hinaus geben sie ausführliche und leicht nachvollziehbare Anregungen für die praktische Arbeit von Lehrenden nicht nur im Schulkontext, sondern auch in der universitären Ausbildung zukünftiger Lehrerinnen und Lehrer. Die Bandbreite der Anregungen und der vorgestellten Projekte reicht u.a. von der Verbindung von Sprache und Musik, über Sprache und Bewegung bis hin zu Sprachenlernen und Kunst.

Zunächst werden die verschiedenen Aspekte des ästhetischen Lernens skizziert, um sich dann den Grundfragen zuzuwenden: Was konstituiert ästhetisches Erleben? und wie kommt kulturelle Bildung zustande? Eingangs wird ein durchaus positives Bild des ästhetischen Status Quo an modernen 
Schulen entworfen: So geht man optimistisch davon aus, dass es an modernen Schulen nicht nur Schulorchester, Chöre, Theater-AGs, Kunstwerkstätten und dergleichen gibt, sondern dass auch für extra-curriculare Arbeitsgruppen externe Expertinnen und Experten, Künstlerinnen und Künstler herangezogen werden. Hier sei kritisch angemerkt, dass dies in Zeiten von „teaching to the test" und leeren Kassen leider eher selten der Fall sein dürfte. Nichtsdestotrotz ist die Forderung nach einem ästhetischen Lernambiente, in dem sich Lernende gut aufgehoben fühlen, sowie nach der Hinzunahme ästhetischer Lehr- und Lernfächer eine berechtigte.

Neben der allgemeinen Beschäftigung mit der Frage, was ästhetische Bildung überhaupt meint, sind die Beträge aus der Praxis das Spannende und Erhellende in diesem Band. So führt der Weg den Leservon Themen wie dramapädagogischer und theaterpädagogischer Bildungsarbeit in der universitären Lehre für angehende Lehrkräfte, über die Verbindung von Sprache und Musik auf Grundlage des neurokognitiven Diskurses weiter zu Sprachförderung durch Rap oder zu sprachlichem und ästhetisch-kulturellem Lernen durch Kunst. Der vorliegende Band kann als Plädoyer für die „Kulturschule“ im Sinne eines kulturell-ästhetischer Lernortes verstanden werden, an dem die Schülerinnen und Schüler mit allen ihren Sinnen lernen. Argumente für ein expliziteres Hinwenden zu dieser Art ganzheitlicher Bildung lassen sich in Zeiten von Integration, Inklusion und Mehrsprachigkeit und den vielen damit einhergehenden Herausforderungen zweifelsohne finden, wie von den Beitragenden anhand der unterschiedlichen Projekte und Forschungen eindrücklich dargestellt wird.

Der rote Faden, der sich durch alle Beiträge zieht, ist, dass Kinder und Jugendliche beide Seiten von Bildung brauchen (das sogenannte Fachwissen, aber auch die Beherrschung der jeweiligen Kulturtechniken), um den Sinn ihres eigenen Denkens, Handelns und Fühlens zu erfahren. Denn erst wenn beides ineinandergreift, werden junge Menschen zu verantwortungsbewussten und mündigen Mitgliedern unserer Gesellschaft. Darüber hinaus wird durch die praxisbasierten Arbeiten der Beitragenden die enge Verknüpfung von Geist, Emotionen und Lernen anschaulich nahegebracht.

Die Autorinnen und Autoren sowie ihre Herausgeberinnen zeigen verschiedene Wege heraus aus der rein kognitiven Ausrichtung des Lernens, die zunehmend den Schulalltag dominiert und doch nicht die gewünschten Ergebnisse erreicht - denken wir nur an die sogenannten „Millenials“, denen oft der Zugang zu den schönen Künsten als mühsam und nicht zielorientiert erscheint (wie oft bekommt man als Lehrperson die Frage gestellt: „Wofür brauche ich das? Ich will doch nicht in die Kunst, Literatur, etc. gehen!") Gemeinsam weisen die Autorinnen und Autoren auf eindrucksvolle Weise nach, dass die Verbindung aus Sprachenlernen und performativen Kunstformen einen hohen pädagogisch-didaktischen Mehrwert bietet. In ihren Beiträgen führen sie aus, wie wichtig und effektiv das Lernen im Prozess aus ko-konstruiertem Wissen und unmittelbarer emotionaler Erfahrung vonstattengeht. So kommen Lesende nicht um die Erkenntnis umhin, dass die Verbindung aus Sprachenlernen und performativen Kunstformen weit mehr ist als bloße Nischendidaktik - 
ausgeführt und präferiert von einigen wenigen. Das Buch fordert heraus, sich auf bisher vielleicht eher unbekanntes Terrain zu wagen: das des miteinander (Theater-)Spielens, das des gemeinsam gestalteten Musizierens, das des künstlerischen Ausgestaltens - ohne dabei jedoch das Ziel des Sprachen- und Kulturlernens aus den Augen zu verlieren.

Exemplarisch lässt sich hier der Beitrag Lehre trifft Theater (Kooperationsprojekt mit dem KRESHTheater Krefeld und dem Institut Deutsch als Zweitsprache/Deutsch als Fremdsprache der Universität Duisburg-Essen) von Anastasia Moraitis heranziehen. Sie erläutert, dass sich weder ästhetisches Lernen noch ästhetische Bildung in eben jenen normativen Kontexten vermitteln lassen, die in unserem Schulsystem, aber auch in der universitären Ausbildung der zukünftigen Lehrerinnen und Lehrer immer noch fest etabliert sind. Auch wenn Lehren häufig als Kunst angesehen wird (vgl. Lutzker 2007) und Lehrende von den vielfältigen Fähigkeiten einer auch theaterpädagogischen Ausbildung profitieren würden, bleiben entsprechende Angebote weiterhin die Ausnahme. Anhand des vorgestellten - und praktisch durchgeführten - Seminarablaufes gelingt es hier zu verdeutlichen, wie das Aufeinandertreffen von Lehre und Theater gestaltet werden kann und welche tiefgreifenden Erfahrungen Lehramtsstudierende auf diese Weise gewinnen können.

Es fällt leicht, sich der Schlussfolgerung anzuschließen, dass sowohl in der universitären Ausbildung als auch in den Schulcurricular Raum und Zeit für neue Methoden und Erfahrungen geschaffen werden muss, wenn holistisches (Sprachen-)Lernen gelingen soll. Bisher fehlt es auf der Schulebene wie auch in der Ausbildung von angehenden Lehrerinnen und Lehrern an emotionaler Einbindung.

Auf jedem Fall motiviert das Buch Lehrende wie Lehramtsstudierende gleichermaßen, sich weiter mit dem Thema zu beschäftigen.

\section{Bibliografie}

Lutzker, Peter (2007): The Art of Foreign Language Teaching. Improvisation and Drama in Teacher Development and Language Learning. Tübingen: Francke Verlag 